\title{
Dépistage urinaire : les limites de l'immunoanalyse
}

\section{Urine screening, limitations of immunoanalysis}

\section{Bernard CAPOLAGHI*}

Laboratoire de Biochimie-Toxicologie, Hôpital Bel-Air, CHR Metz-Thionville - 57100 THIONVILLE

* Auteur à qui adresser la correspondance : Bernard CAPOLAGHI, Laboratoire de Biochimie-Toxicologie, Hôpital Bel-Air, CHR Metz-Thionville - BP 60327 - 57126 THIONVILLE Cedex Tél : 0382558199 - Fax : 0382558201 - e-mail : bcapo@ wanadoo.fr

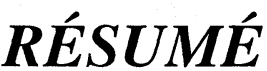

Le dépistage de substances psychoactives est généralement synonyme de méthodes immunologiques et de prélèvement urinaire. Il s'agit d'une part de privilégier un prélèvement biologique non traumatisant et ne nécessitant pas de compétences professionnelles au niveau du recueil et d'autre part de disposer d'une technique facile à utiliser, transposable aisément et automatisable.

Cette adéquation théorique se heurte cependant à de nombreuses contraintes analytiques qui limitent le champ d'application du dépistage urinaire.

Les méthodes immunologiques utilisent toute la reconnaissance épitopique spécifique de la molécule à doser ou du chef de file de la famille à rechercher. Par contre, la méthodologie utilisée peut être très différente tant pour la nature des anticorps utilisés que pour le choix du mode de séparation et de révélation du complexe Ag-Ac et du type de signal généré. Cette hétérogénéité des techniques explique les similitudes et les différences observées dans le dosage ou le dépistage de substances psychoactives au sein d'une matrice biologique comme le milieu urinaire. Cette matrice non protéique peutêtre considérée comme un milieu biologique de choix; cependant, la complexité physiologique dont elle est issue induit de nombreux problèmes liés le plus souvent soit à une dilution trop importante, soit à une concentration exagérée de composés endogènes ou exogènes pouvant interférer sur les réactions immunologiques. Ces différents aspects nécessitent d'identifier clairement les incidences du choix d'une technique d'immunoanalyse en milieu urinaire sur le résultat.

MOTS-CLÉS

Dépistage urinaire, Immunoanalyse, Toxicomanie.

\section{SUMMARY}

Psychoactive drugs' screening usually means immunological methods and urine analysis. The goal is first to propose the use of a non-invasive sample, easy to obtain even for nonprofessional people, and also to perform the analysis with an automated convenient method. In fact, analytical problems limit the validity of urinary screening.

Immunological methods are based on the reaction between a specific epitope and a dedicated antibody. Of course, antibodies can vary in different methods (monoclonal antibodies, polyclonal antibodies,...), as can also be different the ways of detection and the separation principles. This heterogeneity can explain that there are similar or divergent results between all the available methods. If urine can be considered as a first choice biological matrix, it has to be noticed that this fluid can be too concentrated, too diluted, and can also accumulate exogenous or endogenous compounds, able to interfere with the immunological reaction. The consequences are that a perfect knowledge of the analytical performances of the immunological method used is necessary to correctly interpretate the results of a urinary analysis.

\section{KEY-WORDS}

Urinary screening, immunoassay, addiction. 


\section{Introduction}

La mise en évidence d'une conduite addictive en milieu professionnel nécessite une contribution biologique pour la recherche et l'identification des substances psychoactives impliquées. La preuve de la réalité de consommation repose sur des techniques de confirmation après positivité des méthodes de dépistage. L'approche de première intention nécessite d'une part de privilégier un prélèvement biologique non traumatisant, si possible ne nécessitant pas de compétences professionnelles au niveau du recueil, et d'autre part de disposer d'une technique analytique facile à utiliser. La réponse à cette problématique s'est tout naturellement orientée vers un prélèvement urinaire associé à une méthode immunologique.

L'urine est en effet considérée comme le milieu biologique de choix, tant par sa facilité de recueil que par ses caractéristiques physiologiques. Il s'agit en effet d'une humeur biologique contenant sous formes libres et conjuguées les molécules psychoactives et leurs métabolites pendant une durée généralement assez longue, liée à la métabolisation du produit (tableau I) à une concentration dépendante des fonctions rénales et hépatiques du sujet ainsi que de sa corpulence et de son état d'hydratation. Le recueil sera effectué dans un flacon à usage unique sans antiseptique, ni conservateur et conservé à $4^{\circ} \mathrm{C}$. Il convient d'assurer ce recueil dans des conditions strictes d'identification et d'authenticité physiologique afin de respecter l'approche qualité de la phase pré-analytique et de ne pas induire un biais dans le résultat.

D'autre part, il est important de se prémunir contre une dilution ou une adultération de l'urine par le patient (1) en appliquant des règles strictes au moment du recueil et en vérifiant, le cas échéant, l'intégrité du prélèvement par la mesure de la créatininurie, du $\mathrm{pH}$ et de la température. De la qualité du prélèvement dépend en effet le résultat.

\section{L'immunoanalyse}

L'évolution de l'immunoanalyse a permis de disposer de techniques immunologiques faciles à utiliser, transposables et automatisables, compatibles avec une utilisation en routine dans le cadre du dépistage urinaire. Ces méthodes apparemment simples sont en fait très complexes. Elles associent plusieurs étapes pour permettre l'identification du produit (2). Ainsi, après une reconnaissance épitopique spécifique de la molécule ou du chef de file de la famille à rechercher, la méthode utilisée peut être très différente tant pour la nature des anticorps utilisés que pour le choix du mode de séparation et de révélation du complexe Ag-Ac et du type de signal généré.

Parallèlement aux méthodes d'immunoanalyse en milieu liquide par compétition réalisées en phase homogène ou hétérogène (EMIT, FPIA, CEDIA, KIMS, RIA..), de nouveaux tests immunologiques unitaires sur support solide (immunochromatographie) sont apparus sur le marché et complètent l'offre dédiée au dépistage urinaire. Au niveau européen, le projet «Rosita» s'est fixé comme objectif l'inventaire et l'évaluation de ces tests de dépistage (3).

L'hétérogénéité des techniques existantes sur le marché explique les similitudes et les différences observées dans le dosage ou le dépistage de substances psychoactives au sein d'une matrice biologique comme le milieu urinaire. Cette matrice non protéique peut être considérée comme un milieu biologique de choix ; cependant, la complexité physiologique dont elle est issue induit de nombreux problèmes liés le plus souvent soit à une dilution trop importante, soit à une concentration exagérée de composés endogènes ou exogènes pouvant interférer sur les réactions immunologiques.

Tableau I : Élimination urinaire des stupéfiants.

\begin{tabular}{|l|c|c|}
\hline & Seuil de positivité & $\begin{array}{c}\text { Temps d'élimination } \\
\text { (Estimation moyenne) }\end{array}$ \\
\hline Cocaïne & $\begin{array}{c}300 \mu \mathrm{g} / 1 \\
\text { Benzoyl Ecgonine }\end{array}$ \\
\hline Opiacés & $\begin{array}{c}300 \mu \mathrm{g} / 1 \\
\text { Morphine }\end{array}$ \\
\hline Amphétamines & $\begin{array}{c}1000 \mu \mathrm{g} / 1 \\
d \text {-Amphétamine }\end{array}$ \\
\hline Cannabis & $50 \mu \mathrm{g} / \mathrm{l}$ & 3 jours \\
& THC - COOH & $2-4$ jours (fumeur non régulier) \\
\hline
\end{tabular}




\section{Limites de l'immunoanalyse}

Les partenaires de la réaction immunologique, l'antigène traceur et l'anticorps jouent un rôle essentiel qu'il faut bien connaître pour interpréter correctement le résultat d'une immunoanalyse. La nature de l'antigène ainsi que la concentration retenue pour établir un seuil de positivité ont un rôle clé puisque chaque résultat sera interprété en comparant la réponse de cette molécule à ce seuil. La diversité des réponses des différentes molécules d'une même classe vis-à-vis d'un anticorps doit inciter à la prudence dans l'interprétation d'un test immunologique.

La connaissance des limites du dépistage urinaire par immunoanalyse est donc indispensable à son utilisation optimale. Les performances du test utilisé en termes de sensibilité, spécificité et réactions croisées doivent être parfaitement maîtrisées en vue d'une interprétation correcte du résultat. Ces indications sont données par le fournisseur et doivent être connues par le prescripteur, en particulier la nature des principales substances interférentes. La recherche urinaire des opiacés et des amphétamines est sur ce point très significative des erreurs d'interprétation qu'il faut absolument éviter.

Le métabolisme des opiacés conduit à l'élimination urinaire de la morphine libre ou conjuguée. L'anticorps généralement utilisé reconnaît le noyau morphinane. Les urines contenant des molécules à usage thérapeutique : codéine, codéthyline, pholcodine ou des molécules d'usage illicite, 6-monoacetyl-morphine (métabolite de l'héroïne) seront indifféremment reconnues dès que leur concentration sera supérieure au seuil de positivité du test.

A l'inverse, aucun des morphinomimétiques (buprénorphine, méthadone, dextropropoxyphène, tramadol) dont la structure chimique correspond à un noyau morphinane modifié n'entraînera de positivité. Il y a donc lieu d'introduire la notion de présomption et non de certitude de positivité. La recherche d'une meilleure spécificité, c'est-à-dire une diminution du nombre de faux positifs, peut parfois être obtenue au détriment de la sensibilité en augmentant la valeur du seuil de positivité («cutoff»). Ce fut le cas récemment aux Etatsunis où la Substance Abuse and Mental Health Services Administration a relevé de 300 à $2000 \mu \mathrm{g} / 1$ le seuil de positivité des opiacés en milieu professionnel pour réduire les faux positifs liés à la consommation alimentaire des graines de pavot (4).

L'utilisation des trousses «amphétamines» (anticorps mono ou polyclonaux) demande la même prudence d'interprétation (5) car les amines sympathomimétiques, les analogues structuraux à usage thérapeutique courant comme les décongestionants de voies nasales (éphédrine, pseudo-éphédrine, phénylpropanolamine) ou comme les anorexigènes (clobenzorex, fenfluramine peuvent interférer de façon variable. Par ailleurs, l'émergence d'analogues chimiques de l'amphétamine et de la méthamphétamine (designer's drugs) complique également le problème et nécessite lors du dépistage l'emploi de tests plus spécifiques (6).

\section{Conclusion}

L'utilisation de l'immunoanalyse en dépistage urinaire est amplement justifiée dans la mise en évidence de conduites addictives en milieu professionnel.

Cependant, elle nécessite une parfaite connaissance de ces limites pour une utilisation optimale. L'importance des réactions croisées illustre la nécessité d'une démarche de confirmation d'un résultat positif obtenu par une technique immunologique. Il convient d'opter pour une technique basée sur un principe différent de la reconnaissance épitopique et présentant une meilleure spécificité pour une sensibilité au moins équivalente. Les méthodes chromatographiques sont donc toutes indiquées et recommandées pour cette approche analytique complémentaire.

\section{Références}

1. Dumestre-Toulet V., Verstraete A. Les adultérants des tests urinaires. Toxicorama. $1999 ; 3: 155-66$.

2. Masseyeff R. F. Principles of Immunoassays : Classification of methods. In : Methods of Immunological Analysis. VCH, Weinheim : 1993 ; 11533.

3. Samyn N., Areschka V., Verstraete A. Évaluation de tests de dépistage des drogues sur le terrain. Ann. Toxicol. Anal., $2000 ; 2$ : 105-15.

4. Lee P.R, Shahala D.E. Changes to the cutoff levels for opiates for Federal Workplace Drug Testing Programs. Substance Abuse and Mental Health Services Administration. Fed Register. 1995 ; 60 : 575-85.

5. Vertraete A., Van Haute I. Spécificité des immunoessais pour la détection des amphétamines. Toxicorama. 1997 ; $2: 65-71$.

6. Lekskulchai V., Mokkhavesa X. Evaluation of Roche Abusreen Online Amphetamine Immunoassay for Screening of New Amphetamine Analogues. J. Anal. Toxicol. $2001 ; 25: 471-5$. 